\title{
Metabolomics for bioactivity assessment of natural products.
}

\begin{abstract}
Natural products historically have been a rich source of lead molecules in drug discovery, based on their capability to create unique and diverse chemical structures. However, it is also true that the vast number of metabolites typically present in natural products and their huge dynamic range results in the loss of many possibly bioactive natural compounds, becoming an inextricable obstacle for drug development. Recently, new strategies which favour a holistic approach as opposed to the traditional reductionist methods used previously, have been introduced with the purpose of overcoming the bottlenecks in natural product research. This approach is based on the application of new technologies, including metabolomics, for example. Metabolomics allows a systematic study of a complex mixture such as a phytochemical preparation, which can be linked to observations obtained through biological testing systems without the need for isolating active principles. This may put drug discovery from natural products back in the limelight again. In this review paper, the description of some examples of successful metabolomics applications in several important fields related to drug discovery from natural sources aims at raising the potential of metabolomics in reducing the gap between natural products (NP) and modern drug discovery demand.
\end{abstract}

Keyword: Bioactivity; Drug discovery; Metabolomics; Natural product 